\title{
Scheduling Differentiated Traffic in Multicarrier Unlicensed Systems
}

\author{
Giannis F. Marias and Lazaros Merakos \\ Department of Informatics, University of Athens, TK15784, Tel: +30107257560 \\ \{marias@mm.di.uoa.gr, merakos@di.uoa.gr
}

\begin{abstract}
Over the last few years, a number of mechanisms have been proposed for scheduling different type of traffic over base stations-oriented wireless and mobile systems. The majority of these mechanisms focus on access control in the base stations-to-mobile units part of the wireless and mobile system. Recent proposals for the unlicensed spectrum in the $5 \mathrm{GHz}$ band redefine the problem, since base stations operated by different operators and organizations in overlapping geographical areas need access resolution mechanisms to allocate wireless resources. This issue is addressed here, and a multicarrier access control scheme, called QoS based Dynamic Channel Reservation (QDCR), is proposed. QDCR allows base stations to select the least congested available carrier, to compete for carrier reservation based on QoS requirements, and to share the allocated carrier and time with its associated Mobile Terminals (MTs).
\end{abstract}

\section{Introduction}

The current Internet provides only a best effort service, and it is sufficient for traditional Internet applications like web browsing and e-mail. On the other hand, several target applications require better performance than the best-effort Internet. The ATM, the Integrated Services (IntServ) [1] and more recently, the Differentiated Services (DiffServ) architecture [2], although different, can offer services over Internet (or Intranets) that go beyond the best effort. To meet the increasing demand for wireless and mobile multimedia services, future wireless and mobile networks should adopt new technologies and mechanisms in order to provide the high capacity and the QoS required to support broadband services under limited radio spectrum. During the last years, wireless and mobile ATM, and IP capable systems are nominated as the best option to extend wireline Internet or Intranet, in contrast to the traditional circuit switched voice-based solutions.

In the wireless communication area, the FCC has opened a $300 \mathrm{MHz}$ for Unlicensed National Information Infrastructure (U-NII) band within the $5 \mathrm{GHz}$ range. The FCC stated that in making available this spectrum it anticipates that these U-NII devices, which do not require licensing, will support the creation of new wireless services. The U-NII spectrum is allocated at $5.15-5.25 \mathrm{GHz}, 5.25-5.35 \mathrm{GHz}$ and $5.725-5.825 \mathrm{GHz}$ frequency bands, with different transmission power regulations. Devices operating with low power, such as phones and handsets will use the low U-NII band, portable devices on a SOHO environment will use the middle U-NII band, and devices operating over larger coverage areas, such as mobile phones and terminals, will use 
the high U-NII band. On the other hand, in Europe, the $5 \mathrm{GHz}$ band has been reserved by ETSI for the HIPERLAN (High Performance Radio LAN), which is under development within the Broadband Radio Access Network (BRAN) project. Depending on national regulations, $100-150 \mathrm{MHz}$ is reserved, but there are proposals to extend this range up to $445 \mathrm{MHz}$. The HIPERLAN/2 network uses a Convergence Layer, to become fixed network independent; it will be able to provide the Quality of Service (QoS), which users expect from wired IP and ATM networks, with data transfer rates up to 25Mbps [3]. In Japan, the $5.15-5.25 \mathrm{GHz}$ band has been allocated by MPT to high-speed wireless access. From the FCC, ETSI and MPT regulations, it is expected that the license exempt $5 \mathrm{GHz}$ band will accommodate high speed wireless ATM (wATM) and wireless IP (wIP) applications world wide.

Due to the unlicensed characteristic of the $5 \mathrm{GHz}$ band, dynamic carrier allocation mechanisms are essential. These mechanisms should avoid the use of interfered carriers, especially in outdoor environments, where several operators might share the available spectrum in overlapping coverage areas. Fixed allocation method requires prior arrangements between operators, whilst dynamic allocation (e.g., DCA) does not. Moreover, DCA is more suitable than fixed allocation (e.g., FCA) methods for supporting guaranteed $\mathrm{QoS}$ wireless applications [4].

This paper presents a QoS based, Dynamic Channel Reservation (QDCR) architecture, suitable for Base Station (BS) oriented wATM or wIP systems. QDCR provides a set of rules followed by BSs, to regulate competition, and facilitate wireless resource reservation, based on differentiation of the expected level of service. According to QDCR, each BS, through contention periods, dynamically discovers its adjacent or co-channel interference neighbors, and competes with them taking into account differential QoS demands, traffic demands, and perceived QoS. The proposed approach requires no RF survey, and frequency pre-planning phases. Thus, providers do not have to produce interference matrices (e.g., the compatibility matrix in [5]). Moreover, the proposed method is QoS adaptive, since it takes into account the QoS of the established connections and the perceived delays for carrier reservation.

The structure of the paper is as follows. Section II discusses the assumptions that cover the wireless system, in order to apply the QDCR method. In Section III, we present the distributed contention and reservation method, which enables contention, based on service differentiation. In Section IV we describe the simulation environment and illustrate the performance of QDCR, based on the simulation results. Finally, in Section V, we summarize the conclusions.

\section{System Assumptions}

QDCR applies to systems where the BSs act as communication hCF, offering wIP or WATM access to MTs. Each MT maintains an association with one of the BSs, until it performs a handover. MTs do not communicate directly with other MTs (i.e., no adhoc features are assumed). Each BS uses a Medium Access Control (MAC) mechanism and controls the radio access of its associated MTs. The MAC could be based on any dynamic, slotted, Time Division Multiple Access/Time Division Duplex (TDMA/TDD) approach. This MAC includes a time-scheduling mechanism, which schedules the transmission of MPDUs (for both uplink and downlink directions) 
based on QoS requirements per connection and MT, and produces variable length time frames. Each time frame starts with a Frame Header (FH), which includes a slot map. Each associated MT reads this FH in order to determine which uplink or downlink slots in the frame are scheduled for it. The structure of the wireless MAC frame might be similar to [8], even if [8] was introduced for wATM.

We assume that the available unlicensed spectrum $\mathrm{B}$ (from $\mathrm{X}_{0} \mathrm{MHz}$ to $\mathrm{X}_{1} \mathrm{MHz}$ ) is divided into $\mathrm{M}$ broadband carriers, each of $\mathrm{W} \mathrm{MHz}$. The central carrier, $\mathrm{Fc}$, are given by the equation $\mathrm{Fc}=\mathrm{F}_{0}-\mathrm{cW} \mathrm{MHz}$, where $\mathrm{F}_{0}=\mathrm{X}_{1} \mathrm{MHz}$ and $\mathrm{c}=0,1, \ldots, \mathrm{M}$. The spectrum between $\mathrm{Fc}=\mathrm{F}_{0}-\mathrm{W} / 2 \mathrm{MHz}$ and $\mathrm{Fc}=\mathrm{F}_{0}+\mathrm{W} / 2 \mathrm{MHz}$ is the $\mathrm{RF}$ carrier Fc. For $1 \mathrm{bit} / \mathrm{hz}$ modulation efficiency and $25 \mathrm{MHz}$ channelization scheme, a throughput of $25 \mathrm{Mbps}$ per carrier is provided. BSs, belonging to different operators or organizations, using one carrier of the multicarrier structure ( $\mathrm{M}$ carriers) of the unlicensed band $\mathrm{B}$, and, operating in overlapping geographical areas, should first reserve time on one carrier, before starting scheduling the reserved time to their associated MTs (through the MAC protocol). Moreover, in environments where the BSs switch among carriers, it is essential to support a mechanism that informs the MTs about the next carrier that their associated BS will use. For this purpose, we introduce a Frame Trailer (FT), transmitted at the end of the time frame. QDCR assumes that all the components (competing BSs and associated MTs) are slot synchronized, and they use the same TDMA slot length. We assume that a BSs can use one RF carrier at a time (i.e., single antenna assumption). The total number of $M$ carriers is available for all BSs. We further assume that the BSs do not communicate directly, and that all the BSs use the same transmitted power level, PBS, and all the MTs use the same transmitted power level, PMT. Normally PMT $\leq$ PBS. For instance Tx power can be $100 \mathrm{~mW}$ (20dB) for small indoor coverage areas, or $1000 \mathrm{~mW}$ for outdoor larger coverage areas (i.e., HIPERLAN type 2 and U-NII middle band). To sense idle carriers, a threshold of Pth $\mathrm{dBm}$ (e.g. $-100 \mathrm{dBm}$ ) is adopted for the BSs. Furthermore, omnidirectional antennas are considered for the BSs. To cope with Turn Around Times (TAT), that is time required to switch from receive to transmit mode and vice versa, we assume that all MTs require one slot. When BSs communicate with MTs (i.e., during their reservations), they use one slot for TAT; otherwise, BSs TAT is considered smaller (e.g., during competition). Likewise, for the Switch Carrier Time (SCT), that is time required to switch carrier we assume that all MTs require one slot. When BSs communicate with MTs (i.e., during their reservations) they use one slot for SCT; otherwise, BSs SCT is considered smaller (e.g., during competition periods). In indoor installations, wIP or wATM LANs might be isolated, and covered by a single operator. The isolated wLANs do not interfere with another wLAN operating in the same area. In such a case, carrier allocation can be performed dynamically, in a centralized fashion, through a central scheduling entity [9], which co-ordinates the access to the shared wireless resources.

\section{QDCR Mechanism}

Providing QoS services in a mobile environment requires that the radio MAC supports some degree of separation between different types of services. We propose to support three service classes: loss sensitive, delay sensitive and best effort. To 
provide efficient carrier selection criteria, QoS based access, and low reservation delays, QDCR uses special signal bursts broadcast by BSs. Moreover, to avoid congestion, QDCR separates control and data channels. Control channels are used to resolve contentions and to broadcast carrier status information.

\subsection{Burst Signals}

A signal burst is energy transmitted by BSs to indicate certain conditions and to broadcast control information. Burst signals use a higher transmitting power level, BPBS, than normal bursts, i.e., BPBS > PBS. QDCR uses the following burst signals:

- A BS transmits the Priority Burst Signal (PBS) during the priority resolution phase, declaring its QoS demand.

- A BS transmits the Request Burst Signal (RBS), during the competition phase, declaring information such as perceived delay, and reservation period request.

- A BS transmits the Periodic Priority Burst Signal (PPBS) periodically.

PBS requires less than a slot for its transmission The RBS duration is variable. PPBS is broadcast periodically, requires one slot to transmit, and the period is a system parameter.

\subsection{QDCR Channels}

According to QDCR, once one or more BSs sense idle carrier, the QDCR superframe starts. This superframe consists of several periods (control and data channels), allowing BSs to solve the competition, to reserve the carrier, to exchange data with their associated MTs, and to broadcast control information. The QDCR superframe channels are: a) the Priority Resolution Channel, b) the Contention Resolution Channel, c) the MAC Channel, and d) the CF Channel.

Priority Resolution Channel (PR-CH) - During this period, each BS estimates its Reservation Priority (RP). In QDCR, each BS competes with interferers in order to reserve a carrier for a time period equal to its TDMA time frame. Assume that a $\mathrm{BS}_{\mathrm{i}}$ serves $\mathrm{K}_{\mathrm{i}}$ connections, among all its associated MTs, classified as:

- $\quad\left\{\mathrm{C} 1, \mathrm{C} 2, \ldots, \mathrm{Ck}_{\mathrm{x}}\right\}$ real time connections

- $\quad\left\{\mathrm{V} 1, \mathrm{~V} 2, \ldots, \mathrm{Vk}_{\mathrm{y}}\right\}$ non real time connections

where, $\mathrm{k}_{\mathrm{x}}+\mathrm{k}_{\mathrm{y}}=\mathrm{K}_{\mathrm{i}}$. Real time $(r t)$ connections impose transfer delay requirements, whilst non real time $(n r t)$ connections impose loss requirements. Each $r t$ connection $\mathrm{Ci}\left(0<\mathrm{i}<\mathrm{k}_{\mathrm{x}}+1\right)$ introduces a transfer delay violation threshold, $\mathrm{Di}^{\text {thr }}$. Each $n r t$ connection $\mathrm{Vi}\left(0<\mathrm{i}<\mathrm{k}_{\mathrm{y}}+1\right)$ introduces a cell loss violation threshold, $\mathrm{Li}^{\mathrm{thr}}$. The $\mathrm{RP}$ for a $\mathrm{BS}_{\mathrm{i}}$ is identical to the parameter defined in [10], and given by the following equation :

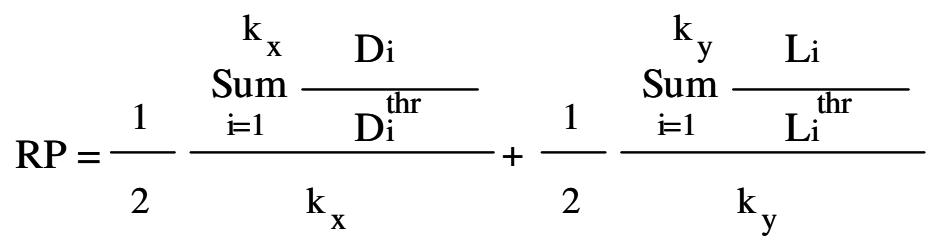

where $\mathrm{Di}$ is the delay that the connection $\mathrm{Ci}$ experiences, and the $\mathrm{Li}$ is the cell loss that the connection $\mathrm{Ci}$ experiences. The PR-CH period consists of a constant number of slots (e.g., 2 slots). This period is further divided to PR-CH minislots (p-slots). Each p-slot position corresponds to a particular RP. For instance assuming a $5 \mathrm{p}$-slot 


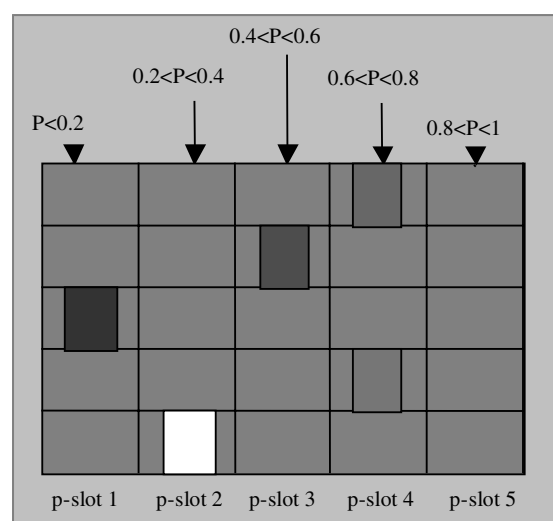

Fig. 1. The BSs broadcast their priorities through PBS bursts on the corresponding p-slot of the PR-CH. The wining BSs broadcaston the higher order p-slots granularity, the first p-slot of the PR-CH period corresponds to $\mathrm{RP} \leq 0.2$, the second p-slot corresponds to $0.2<\mathrm{RP} \leq 0.4$, and the last p-slot corresponds to $0.8<\mathrm{RP} \leq 1$, as shown in figure 1.

According to the estimated $\mathrm{RP}_{\mathrm{i}}$, the $\mathrm{BS}_{\mathrm{i}}$ will broadcast its $\mathrm{PBS}_{\mathrm{i}}$ within the corresponding p-slot. Best effort service class will use the first p-slot of the PR$\mathrm{CH}$ period to broadcast the corresponding PBS. If $\mathrm{D}_{\mathrm{PBS}}$ is the duration of signal PBS, and $D_{P S}$ is the duration of p-slot, then $\mathrm{D}_{\mathrm{PS}}>\mathrm{D}_{\mathrm{PBS}}$, and $\mathrm{TAT}<\mathrm{D}_{\mathrm{PS}}-\mathrm{D}_{\mathrm{PBS}}$. This allows BS to switch from transmit to receive mode and sense PBS broadcast on the next order p-slot. A backlogged BS, i.e., with low PR, sense the PBS burst of the BS illustrating higher PR, because the

latter will broadcast its PBS using a higher order p-slot. Backlogged BSs should select new carrier, among the $\mathrm{M}$ candidates, to compete for it.

Contention Resolution Channel (CR-CH) - On the PR-CH channel we have adopted a structure (i.e., number of p-slots) to represent RPs with certain granularity. Thus, it is possible for two more BS to use p-slots of the same order to broadcast their PBSs, even if their RPs have different values (e.g., on the $2^{\text {nd }}$ decimal digit of RPs). To overcome this problem we introduce the CR-CH. During CR-CH period each BS, survived from priority resolution phase, broadcast its reservation requests (through the RBS), and realizes the reservation requests of other interfering survivors. Reservation requests represent either current MAC frame time length, or mean reservation delay, or both. The CR-CH comprises of an integer, but not fixed, number of slots, each of which is divided to a fixed number of minislots (c-slots), as figure 2 illustrates. The RBS signals are transmitted on continuous c-slots, and simultaneously by the competing BSs. We introduce a granularity factor $\mathrm{g}, 0<\mathrm{g}<1$. If $\mathrm{T}$ slots is the reservation request (MAC length, delay, or both) in slots, then RBS will use [ $\mathrm{g} * \mathrm{~T}] \mathrm{c}$ slots for its transmission. If $\mathrm{D}_{\mathrm{CS}}$ is the duration of c-slot, then $\mathrm{TAT}<\mathrm{D}_{\mathrm{CS}}$. This allows BS to switch from transmit to receive mode and sense RBS broadcast by another BS.

Longest Job First (LJF). According to this discipline the winner of the competition is the BS with the larger reservation request (i.e., having the larger MAC frame). Thus, if $\mathrm{TFD}_{\mathrm{i}}$ is the number of slots the $\mathrm{BS}_{\mathrm{i}}$ wishes to reserve on this carrier (i.e., current time frame length), then the $\mathrm{BS}_{\mathrm{i}}$ transmits a $\mathrm{RBS}_{\mathrm{i}}$ of $\left[\mathrm{g} *\left(\mathrm{TFD}_{\mathrm{i}}\right)\right]$ c-slots. The winner (survivor) BS is the one that broadcasts the larger RBS

Delayed Job First (DJF). The winner of the competition is the BS that received the highest mean delay from its previous reservation attempts on any carrier. Each $\mathrm{BS}_{\mathrm{i}}$ records the last reserved slot in any carrier, say $\mathrm{T}_{\mathrm{Ri}}$, and switches to a carrier in order to compete for it. Then it calculates the $\mathrm{T}_{\mathrm{M}}=\operatorname{mean}\left(\mathrm{T}_{\mathrm{Ri}}\right)$. If the $\mathrm{BS}_{\mathrm{i}}$ is involved in the competition, $\mathrm{BS}_{\mathrm{i}}$ transmits a $\mathrm{RBS}_{\mathrm{i}}$ signal, equal to $\left[\mathrm{g} *\left(\mathrm{~T}_{\mathrm{M}}\right)\right.$ ] c-slots. The mechanism is identical for the LJF and DJF disciplines. In the former case the RBS is proportional 


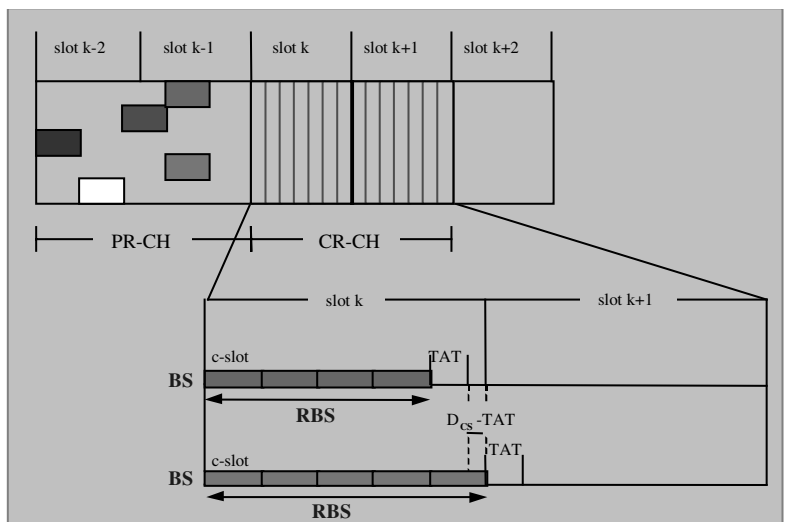

Fig. 2. The Contention Resolution (CR-CH) structure

to the frame size, whist in the latter case the RBS is proportional to the received reservation delay.

Delayed and Longest Job First (DLJF). This is a combination of LJF and DLF disciplines. The winner of the competition is the $\mathrm{BS}$ that experiences the larger combined reservation delay, and time frame. Thus, if $\mathrm{T}_{\mathrm{Ri}}$ is the last reserved slot of a $\mathrm{BS}_{\mathrm{i}}$ in any carrier, and the contention for a carrier involves the $\mathrm{BS}_{\mathrm{i}}$, and $\mathrm{TFD}_{\mathrm{i}}$ is the number of slots the $\mathrm{BS}_{\mathrm{i}}$ wishes to reserve on this carrier (i.e., current time frame length), then the $\mathrm{BS}_{\mathrm{i}}$ transmits a $\mathrm{RBS}_{\mathrm{i}}$ of $\mathrm{g}^{*}\left(\mathrm{TFD}_{\mathrm{i}}+\mathrm{T}_{\mathrm{M}}\right)$ c-slots, where $\mathrm{T}_{\mathrm{M}}=$ mean $\left(\mathrm{T}_{\mathrm{Ri}}\right)$. A backlogged $\mathrm{BS}$, i.e., with low reservation request, sense the RBS burst of the $\mathrm{BS}$ with higher reservation request, because the latter will broadcast an RBS using at least on more cslot. Backlogged BSs should select new carrier, among the M candidates, to compete for it. The survivor is the BS that has completed its RBS transmission, switched on receive mode and senses the carrier idle. This BS will reserve the carrier.

Medium Access Control Channel (MAC-CH) - This period is used for data transfer, i.e., accommodates the MAC frame. It consist of

- Frame Header Broadcast Channel (FHB-CH), Within this channel the BS broadcast its MAC frame slot map to its associated MTs. Moreover, each BS, broadcasts a keyword (as a unique identifier) within the FH This key is exchanged between BSs and MTs during the association phase, and it is used by the MTs in order to identify if the winner of a competition on a carrier is their associated BS.

- Down Link Data Channel (DLD-CH), with variable duration, accommodating information sent through BS to MTs.

- Turnaround Channel (T-CH), which occupies one slot and allows MTs or BSs to switch from receive to transmit mode and vice versa. It is used more than one time per QDCR superframe.

- Up Link Data Channel (ULD-CH), with variable duration, accommodating information sent from MTs to other MTs or fixed terminals through the BS.

- MTs Contention Channel (MC-CH), which allows associated MTs, with no allocated ULD-CH slots, to request reservation slots, or accommodates registration or re-association requests from MTs performing registration or handover. The number of MC-CH slots can be static or dynamic.

- Frame Trailer Channel (FT-CH), which occupies one slot. Within FT-CH the BS broadcast the FT to the associated MTs. FT includes a visiting list of the carriers that the BS will visit sequentially until a successful reservation. The construction of the visiting list is based on Selection Parameters, discussed later..

Periodic Priority Resolution Channel (PPR-CH) - This channel uses one slot, and during this period the BSs broadcast their priorities. This channel is similar to PR-CH 
of the competition period. The difference is that only the sensing BSs broadcast their priorities; the BSs that already have carrier reservation during PPR-CH do not broadcast their priorities, and remain silent. All the BSs know that the PPR-CH signals are broadcast every period of $\mathrm{R}$ slots. Figure 3 illustrates the QDCR superframe and its channels.

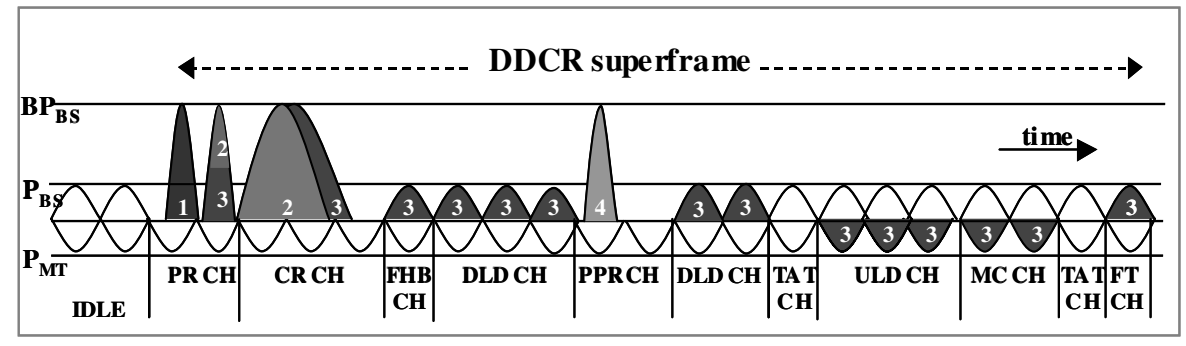

Fig. 3. The channels of the QDCR superframe. In the figure, three mutual interfering BSs compete for one carrier.

\subsection{QDCR Carrier Selection}

Each BS maintains and constantly updates the values of a parameter list named Congestion Factor (CF). This parameter indicates the number of BS's neighbors that compete or use each carrier during a recent period The notation $\mathrm{CF}_{\mathrm{i}, \mathrm{r}}$ denotes the $\mathrm{CF}$ maintained by the $\mathrm{BS}_{\mathrm{i}}$ for carrier $\mathrm{F}_{\mathrm{r}}(0 \leq \mathrm{r}<\mathrm{M})$. $\mathrm{CF}_{\mathrm{i}, \mathrm{r}}$ is updated according the rules:

1. When $B S_{i}$ selects a carrier $F_{r}$ it sets $C F_{i, r}=1$.

2. When $B S_{i}$ realizes that $F_{r}$ is reserved by another $B S$ it sets $C F_{i, r}:=C F_{i, r}+1$.

3. It is possible for a $\mathrm{BS}_{\mathrm{i}}$, listening on a carrier $\mathrm{F}_{\mathrm{r}}$, to sense two or more signals bursts, which are transmitted simultaneously on $\mathrm{p}$ or $\mathrm{c}$ minislots by two or more $\mathrm{BSs}$ using the carrier $\mathrm{F}_{\mathrm{r}}$. In such case, the $\mathrm{BS}_{\mathrm{i}}$ may not be able to receive useful information due to congestion, and it sets $\mathrm{CF}_{\mathrm{i}, \mathrm{r}}:=\mathrm{CF}_{\mathrm{i}, \mathrm{r}}+2$.

4. If during PR-CH or PPR-CH on a carrier $\mathrm{F}_{\mathrm{r}}, \mathrm{R}$ PBS signals are broadcast on $\mathrm{p}$ slots of different order of the p-slot used by $\mathrm{BS}_{\mathrm{i}}$, if $\mathrm{R}_{\mathrm{x}}$ of them are the only PBS signals per $\mathrm{p}$-slot then $\mathrm{CF}_{\mathrm{i}, \mathrm{r}}:=\mathrm{CF}_{\mathrm{i}, \mathrm{r}}+\mathrm{R}_{\mathrm{x}}+2 *\left(\mathrm{R}-\mathrm{R}_{\mathrm{x}}\right)$, according to rule 3 .

5. If $\mathrm{BS}_{\mathrm{i}}$ loses a competition for carrier $\mathrm{F}_{\mathrm{r}}$, it sets $\mathrm{CF}_{\mathrm{i}, \mathrm{r}}:=\mathrm{CF}_{\mathrm{i}, \mathrm{r}}+2$ if for at least one cslot receives useless information due to congestion, otherwise, it sets it sets $\mathrm{CF}_{\mathrm{i}, \mathrm{r}}:=\mathrm{CF}_{\mathrm{i}, \mathrm{r}}+1$.

When choosing a carrier, the BS should choose the carrier illustrating the less congestion. $\mathrm{A} \mathrm{BS}_{\mathrm{i}}$ keeps its Selection Parameters $\left(\mathrm{SP}_{\mathrm{i}}\right)$ list, as follows:

$$
S P_{i, r}=\left(\text { CurrentTime }_{i} \text { LastVisitTime }_{i, r}+1\right) / C F_{i, r} \quad 0 \leq r<M \text {, }
$$

$\mathrm{A} \mathrm{BS}_{\mathrm{I}}$ selects the carrier illustrating the minimum $\mathrm{SP}_{\mathrm{i}, \mathrm{r}}$ value. The Current Time factor represents the time a BS takes a carrier selection decision. The Last Visit Time factor represents the last time the $\mathrm{BS}_{\mathrm{i}}$ was using the carrier $\mathrm{F}_{\mathrm{r}}$. The rate of $\mathrm{CF}$, diminishes in value with time, because the CF represents the congestion, i.e., the number of BSs, on a carrier during a recent time period. Thus, an ageing threshold is used to represent the uncertainty of the congestion for a carrier visited in the distant past. We introduce the following threshold:

CurrentTime-LastVisitTime $_{i, r}>M *$ MeanFrameSize $e_{i} \quad 0 \leq r<M$ 
A $\mathrm{BS}_{\mathrm{i}}$ checks the time elapsed from the latest time the carrier $\mathrm{F}_{\mathrm{r}}$ was used. If for a carrier $F_{r}$ the ageing threshold is exceeded, the $\mathrm{BS}_{\mathrm{i}}$ sets the $\mathrm{CF}_{\mathrm{i}, \mathrm{r}}$ to a predefined value. Different predefined values can be applied. Results presented in [11] illustrate that the best policy for the predefined value, when the ageing threshold of $\mathrm{BSi}$ for the carrier $\mathrm{F}_{\mathrm{r}}$ is violated, is to set the $\mathrm{CF}_{\mathrm{i}, \mathrm{r}}$ to a value that is the lowest of the existing $\mathrm{CF}_{\mathrm{i}, \mathrm{k}}$ values, i.e., $\mathrm{CF}_{\mathrm{i}, \mathrm{r}}=\min \left(\mathrm{CF}_{\mathrm{i}, \mathrm{k}}\right), \mathrm{k}<\mathrm{M}, \mathrm{k} \neq \mathrm{r}$.

\section{Simulation Environment and Results}

To evaluate the QDCR performance, simulations were performed using the OPNET Simulator [12]. The carrier speed was set to 20Mbits/sec, and each TDMA slot was set to 54 bytes long. Each TDMA slot accommodates one MPDU. Each TDMA time frame was assumed to contain 5 slots for control information, i.e., for FH-CH, FT$\mathrm{CH}$, TAT-CH, and MC-CH. For the simulations, we used a combination of both real time $(r t)$ and non real time $(n r t)$ connections. $r t$ connections classified to constant rate $r t(c r t)$, and variable rate $r t$ connections ( $v r t)$. Each $c r t$ connection is simulated by a periodic MPDU generator. We assumed 64Kbps crt connections. For a $20 \mathrm{Mb} / \mathrm{sec}$ channel, each $64 \mathrm{kbps}$ crt connection produces one MPDU every 260 time slots, approximately. We used 50 crt sources (25 uplink and 25 downlink) per BS. On the other hand, each vrt or $n r t$ source is modeled by a Discrete time Batch Markov Arrival Process (D-BMAP). For such a process we consider the TDMA slot as the time unit. According to [13], the traffic load produced by a source can be approximated by the super-position of $U$ equivalent ON/OFF minisources. For each vrt or $n r t$ source, $\mathrm{m}$ was set to $256 \mathrm{Kbps}$ and $\mathrm{s}^{2}$ was set to $128 \mathrm{Kbps}$, where $\mathrm{m}$ and $\mathrm{s}^{2}$ are the mean and the variance of the transmission rate, respectively, whilst the parameter U was set to 10 [13]. For the rt connections (i.e., crt and vrt) the MPDU Transfer Delay (MTD) was set to 50 time slots (i.e., approximately $1 \mathrm{msec}$ ), and the $\mathrm{Di}^{\text {th }}$ (eq. 1) parameter was set to $10^{-6}$. For the $n r t$ connections the MTD was set to 100 time slots (i.e., $2 \mathrm{msec}$ ), and the $\mathrm{Li}^{\text {th }}$ was set to $10^{-6}$. Each $\mathrm{BS}$ assumed to contain a cumulative buffer of 200 MPDUs. We considered two different load classes, load class 1 and 2. For load class 1 each BS accommodates $10 \mathrm{vrt}, 10 \mathrm{nrt}$, and $30 \mathrm{crt}$ connections. For lad class 2 each BS accommodates $20 \mathrm{vrt}, 20 \mathrm{nrt}$, and $30 \mathrm{crt}$ connections. The simulation duration was set to 7 days (approximately for $2 * 10^{10}$ TDMA time slots). For the QDCR mechanism, overheads are due to control channels (PR-CH, CR-CH, PPR-CH). In the simulations we have used two slots for each of the PR-CH and PPR-CH, with 10 p-slots in total, corresponding to 10 discrete priority reservation values. On the other hand, $\mathrm{CR}-\mathrm{CH}$ resolves contention with $5 \mathrm{c}$-slots per contention slot, and a granularity factor $(\mathrm{g})$ of 0.1 . To simulate the interference environment we assumed that all the activated BSs are interfere mutually. QDCR mechanism was compared with alternative carrier selection mechanisms such as Random Choice (RC) and Round Robin (RR). In RC the BS selects randomly one carrier to compete for its reservation. In RR, once the BS starts its operation, it randomly selects a carrier $\mathrm{F}_{\mathrm{r}}(0 \leq \mathrm{r}<\mathrm{M})$ to compete for. In the next selection, the $\mathrm{BS}$ chooses the next carrier (i.e., $\mathrm{F}_{\mathrm{r}+1}$, if $\mathrm{r}<\mathrm{M}-1$, or $\mathrm{F}_{0}$ otherwise). We have performed simulations using different combinations of operating BSs $(\mathrm{N})$ and available carriers (M). Figures 4, 5, and 6 illustrate the mean reservation delay (in time slots) when 8 
BSs compete to reserve 4 available carriers, for LJF, LDJF and DJF competing disciplines, when load class 1 is used. For QDCR it is assumed that PPR-CH is every 20 slots (parameter R).

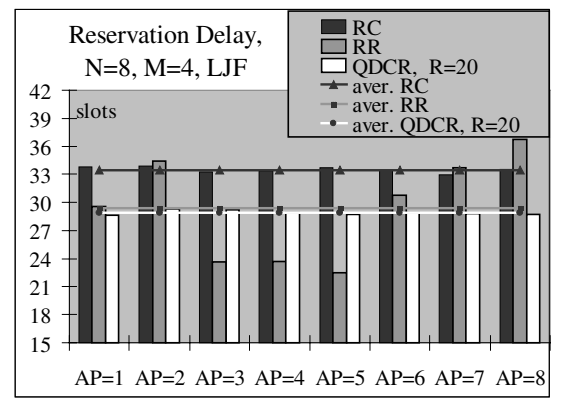

Fig. 4. Reservation delay of the LJF, for RR, $\mathrm{RC}$, and QDCR (load class=1).

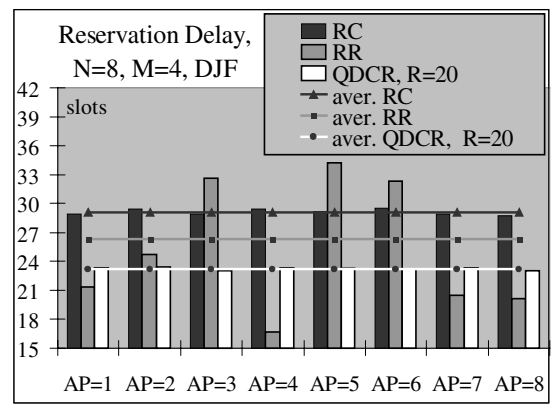

Fig. 5 Reservation delay of the DJF, for $\mathrm{RR}, \mathrm{RC}$, and QDCR (load class=1).

Figures 4, 5, and 6 illustrate that the DJF discipline achieves the lower reservation delays among all the alternative disciplines. QDCR achieves lower reservation delays, than the RC and the RR disciplines. Furthermore, even if the averaged, among the activated BSs, reservation delay for QDCR and RR are almost equal, the QDCR can guarantee fair reservation delays for the operational BSs, whilst RR does not. These observations are valid for all the contention disciplines. During the simulations we have observed that the QDCR mechanism achieves 20-40\% lower reservation delays, than the RC or the RR disciplines, depending on the operational BSs $(\mathrm{N})$, the available carriers (M), and the offered traffic load per BS. Figure 7 compares the performances of the LJF, DJF and LDJF disciplines. The better performance of the DJF, in terms of reservation delay, was confirmed for load class 2 , as well.

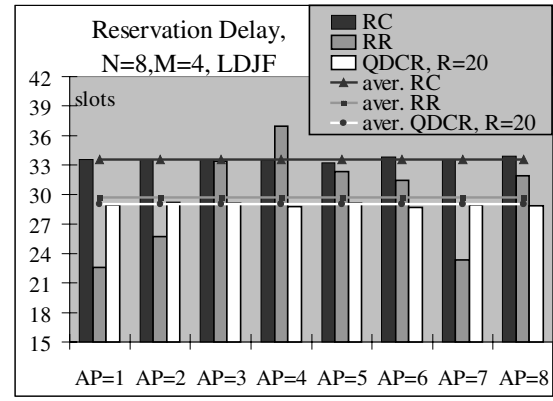

Fig. 6. Reservation delay of the LDJF, for $\mathrm{RR}, \mathrm{RC}$, and QDCR (load class=1).

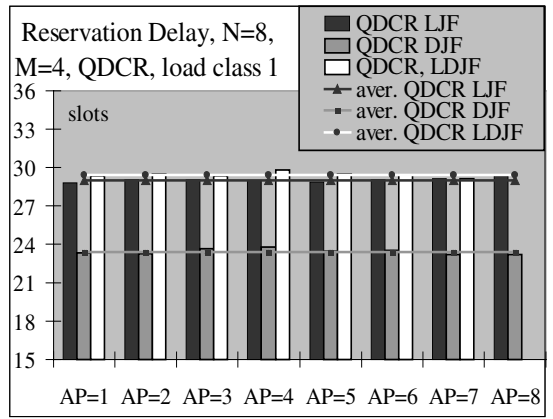

Fig. 7. Comparison of LJF, DJF, LDJF, (QDCR, load class=1).

Another parameter measured in the simulations is the success when predicting carriers' congestion levels. The CF, and the SP parameter lists, as well as the PPR$\mathrm{CH}$, are used by BSs to estimate the congestion level on carriers. Each BS attempts to predict carriers congestion levels when it selects a carrier to compete for; that is, at the end of a reservation, when losing on a competition, or when it realizes, through 
PPR-CH, that another BS with higher priority is sensing the same carrier. Thus, success is the case where by, the carrier selected by the BS accommodates a minimum number of BSs compared to other carriers containing an equal or larger number of BSs at the same time.

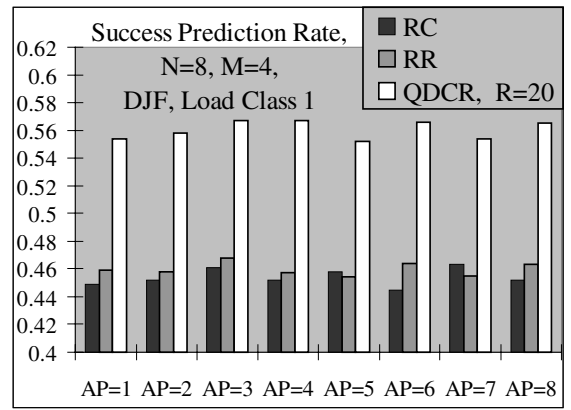

Fig. 8. Success prediction rate for $\mathrm{RR}, \mathrm{RC}$ and QDCR (load class $=1, \mathrm{M}=4, \mathrm{~N}=8$ )

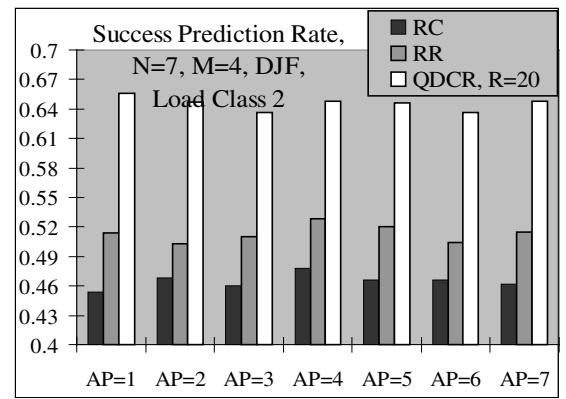

Fig. 9. Success prediction rate for RR, RC and QDCR (load class=2, M=4 N=7)

Figures 8, and 9 illustrate the prediction success rate of the QDCR, RR, and RC methods, for load class $1(\mathrm{~N}=8, \mathrm{M}=4)$, and $2(\mathrm{~N}=7, \mathrm{M}=4)$, respectively, when DJF competition discipline is used. For the QDCR, we have used R=20 (the PPR-CH is repeated every 20 slots). As figures 8 and 9 depict, the QDCR method achieves to predict carriers congestion levels with accuracy of 55\% (load class 1), and 65\% (load class 2). This difference is expected, since we have used the same value for $\mathrm{R}$ parameter $(\mathrm{R}=20)$ for both load classes. The mean TDMA frame size is equal to 15.2, and 50.8 slots, for load class 1 , and 2, respectively; thus, for load class 1 the carriers' status changes more dynamically, than for load class 2 . Setting $R=20$, for load class 1 , the PPR-CH are repeated every two TMDA frames, whilst, for load class 2, the PPR$\mathrm{CH}$ are repeated twice within one TDMA frame. QDCR achieves better success prediction rates for load class 2, because the BSs could switch carrier more times per frame, in a steadier environment, than in the case of load class 1. QDCR can achieve better prediction results, if period $\mathrm{R}$ is low, but there is a tradeoff between overheads (due to control information introduced to achieve higher success rate), and the reservation delay. When $\mathrm{R}=20$ we observed that the overheads due to PPR-CH are $5 \%$, thus QDCR is expected to introduce 5\% more control information, than RR or $\mathrm{RC}$ disciplines, increasing the reservation delay by $5 \%$. The buffer management policy that we have used is as follows. In every slot, each BS checks for MTD violations, and drops $c r t$, vrt, and $n r t$ MPDUs, in this order. If new MPDUs arrived, we time-tag them, and then if the buffer can accommodate these MPDUs, we place these MPDUs in the buffer. Otherwise, we drop from the buffer an equal number of aged MPDUs, in order to accommodate the new load. Concerning the buffer dropping policy, we first drop the older $n r t, v r t$, and $c r t$ MPDUs, in this order. This policy was used for all the set of the simulations. For $\mathrm{N}=8$ operational $\mathrm{BSs}, \mathrm{M}=4$ available carriers, and for load class 1 , we have observed that there are no buffer overflow conditions for the QDCR, RC or RR disciplines. This is due to the buffer management policy, since the algorithm drops MTD violated MPDUs first. On the other hand, we have observed $c r t$ and $v r t$ MTD violations, illustrated in figures 10 and 11. From these figures it is concluded that QDCR produces low dropping ratios of $r t$ MTD violated 
MPDUs, achieving a reasonable MPDU loss ratio, equal $5^{*} 10^{-5}$, for both $c r t$ and $c r t$ type of connections.

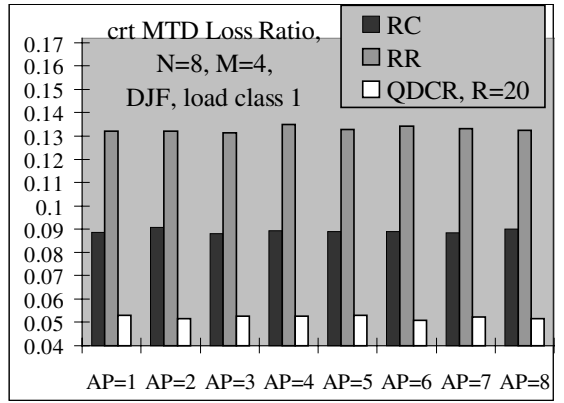

Fig. 10. crt MPDUs Loss Ratio due to MPDU Delay violations (load class 1)

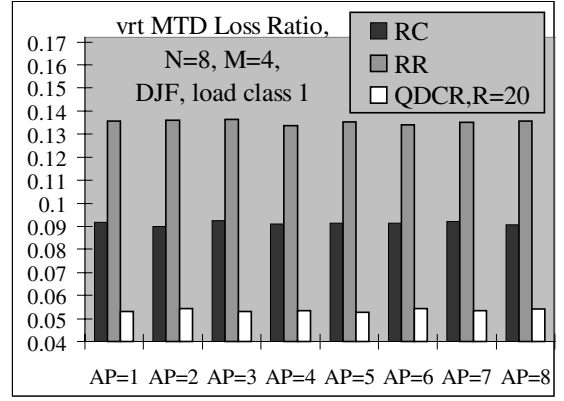

Fig. 11. vrt MPDUs Loss Ratio due to MPDU Delay violations (load class 1)

For $\mathrm{N}=7 \mathrm{BSs}, \mathrm{M}=4$ carriers, and load class 2, figures 12, 13, and 14 illustrate $\mathrm{crt}$, vrt, and $n r t$ MPDUs Loss Ratio, respectively, due to MTD violations, for QDCR, RC, and RR methods, whilst figure 15 depicts the cumulative rt MPDUs Loss Ratio.. Figures 12, 13, 14 and 15 illustrate that QDCR achieves lower MPDU losses on the contenting environment of the load class 2, than the disciplines RR or RC. Even if the MTD thresholds are not satisfied, due to the absence of a connection admission policy, QDCR achieves fairness among the competing BSs, concerning the MTD

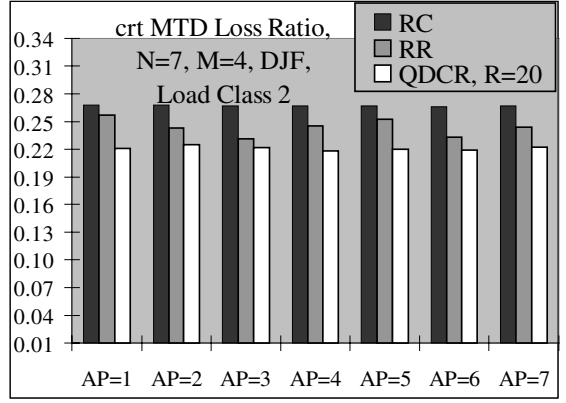

Fig. 12. crt MPDUs Loss Ratio due to MPDU Delay violations (load class 2)

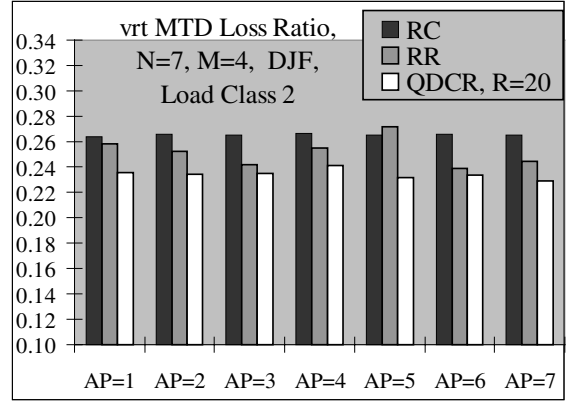

Fig. 13. vrt MPDUs Loss Ratio due to MPDU Delay violations (load class 2)

Figure 16 depicts the MPDU dropping ratio due to buffer overflow conditions, for all the types of the connections. Figure 16 shows that QDCR produces low dropping ratios due to buffer overflow, achieving a reasonable MPDU loss ratio, less than $3^{*}$ $10^{-6}$, for all the types of all kinds of MTs connections. In figure 17 the sum of MPDU losses is illustrated. Figure 17 takes into account all the types of losses (Transfer Delay Violations, Buffer Overflows) for all kinds of connections. We can observe that the QDCR schedules the heavy traffic in a fair and more resourceful fashion, producing lower dropping ratios than RR or RC disciplines. 


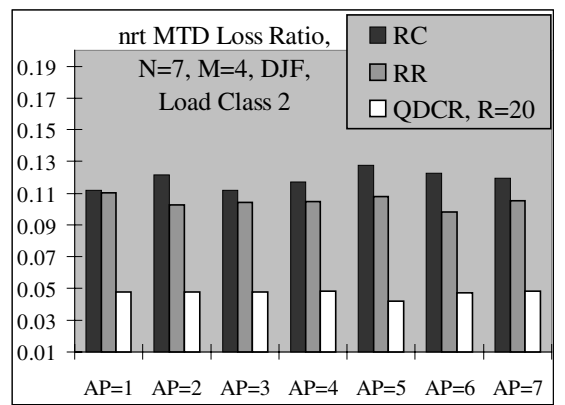

Fig. 14. $\mathrm{n} r t$ MPDUs Loss Ratio due to MPDU Delay violations (load class 2)

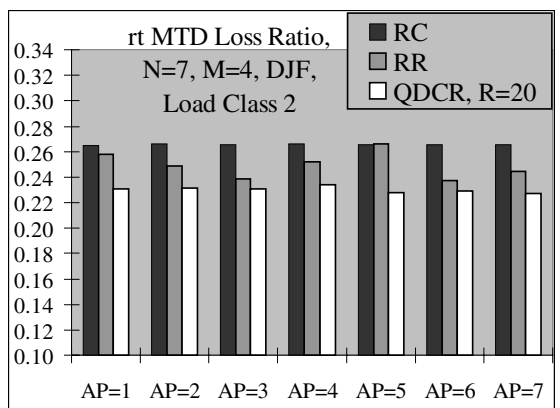

Fig. 15. $r t$ MPDUs Loss Ratio due to MPDU Delay violations (load class 2)

\section{Concluding Remarks}

We have introduced QoS based competition rules for a distributed reservation method, which applies to interfering ATM or IP capable BSs competing for reservation in an unlicensed multicarrier wireless environment. The introduced QDCR mechanism takes into account the differentiated level of service required by the MTs in order to apply a priority based resource reservation method. Moreover, based on real time measurements of carriers' congestion levels, the QDCR mechanism assists BSs to select the least congested carrier in order to compete for its reservation. The QDCR mechanism is immune to topology changes, does not increase power consumption on MTs, and, finally, requires no frequency preplanning.. Furthermore, QDCR imposes no limit on the number of BSs operating in a common area, or on the number of neighboring BSs. The QDCR mechanism achieves to allocate shared resources efficiently. In order to be more virtue for the level of offered service to various types of connections, with different requirements in terms of loss and delay, the QDCR mechanism should be combined with a distributed wireless CAC. The latter could take into account the QDCR decisions, determine if the system is under heavy load conditions, and regulate the admission policy, accordingly.

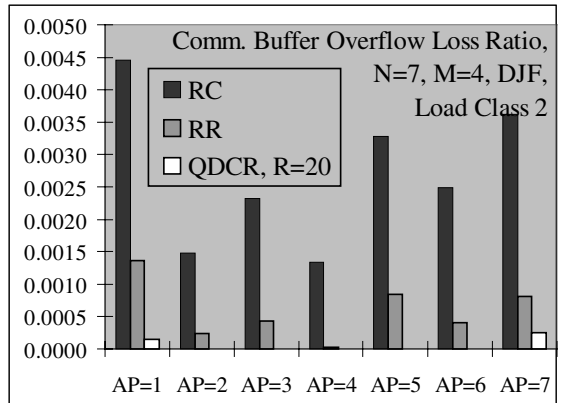

Fig. 16. Loss Ratio due to Buffer Overflow (load class 2)

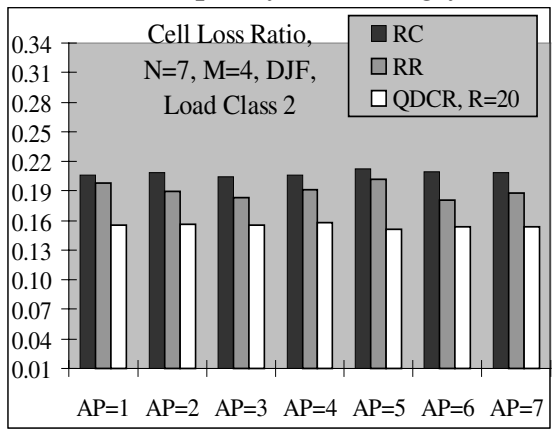

Fig. 17. Loss Ratio due to MTD and Buffer Overflow (load class 2) 


\section{References}

[1] Braden, R., Clark, D., Shenker, S., "Integrated Services in the Internet Architecture: An Overview", IETF RFC 1633, 1994.

[2] Blake, S., Black, D., Carlson, M., Davies, E., Wang, Zh., Weiss, W., "An architecture for Differentiated Services", IETF RFC 2475, 1998.

[3] ETSI, "High Performance Radio Local Area Network (HIPERLAN) Type 2; Requirement and architectures for wireless broadband access", TR 101-031, 1999

[4] V. Li, and X. Qiu, "Personal communications systems," Proc. of the IEEE, Sept. '95

[5] M. Frodigh, "Bounds on the performance of DCA algorithms in highway microcellular systems," IEEE Trans. Vehic. Tech., vol. 43, no. 3, Aug. 1994.

[6] Heinanen, J., et. al., “Assured Forwarding PHB group”, RFC 2597, 1999.

[7] H. Jacobson, V. Nichols, K. Poduri, "An Expedited Forwarding PHB”, RFC 2598, 1999.

[8] N. Passas, L. Merakos, S. Paskalis, D. Vali, "Quality-of-Service-Oriented medium access control for wireless ATM networks" November 1997 issue of IEEE Comm. Mag.

[9] G. F. Marias, D. Skyrianoglou, and L. Merakos, "A Centralized Approach to Dynamic Channel Assignment in Wireless LANs," Proc. IEEE INFOCOM99, NY, USA, Mar. 1999.

[10] ACTS Project, "The Magic WAND", Deliverable 3D6, "Wand DCA Scheme," CEC Del. AC085/INT/ACT/DS/P/034/b1, Aug. 1998.

[11] G. F. Marias, and L. Merakos, "Performance Estimation of a Decentralized Mutlicarrier Access Framework In Unlicensed Wireless Systems," Proc. ICC2001, June 2001, Finland.

[12] OPNET Modeler, MIL 3, Inc., 1993.

[13] C. Blondia, and O. Casals, "Performance analysis of statistical multiplexing of VBR sources," Proc. INFOCOM '92. 